\title{
General and Specific Prevention Strategies for Gastrointestinal Cancers
}

\author{
Peter Malfertheiner \\ Department of Gastroenterology, Hepatology and Infectious Diseases, Otto von Guericke \\ University of Magdeburg, Magdeburg, Germany
}

All initiatives for health maintenance promoted by medical care professionals, medical institutions and societies with involvement of media and broadcastings start with general recommendations on healthy lifestyle (no smoking), nutrition, and physical activity. These efforts have provided good evidence for beneficial effects on human health, but need to be complemented by specific organ-targeted prevention strategies.

For maintenance of digestive health a series of specific strategies have been developed. Non-invasive and invasive screening procedures have proven to significantly reduce the risk of morbidity and mortality in gastric cancer and colorectal cancer, but are less effective or still missing in esophageal cancer.

This issue of Gastrointestinal Tumors presents a selection of small concise reviews updating on the current knowledge and trends in the prevention of malignant diseases of the esophagus, stomach, colon and liver.

Successful prevention is facilitated by the identification of specific etiological factors as either inducers of or contributors to the carcinogenesis in the respective organs and of welldefined precursor lesions of malignancies. Most malignant diseases in the gastrointestinal tract, apart from few inherited genetically driven cancers, have a multifactorial background. Only few neoplasias, such as gastric cancer, have an established principal risk factor, and in the specific case of Helicobacter pylori a screen and treat strategy allows a direct targeted prevention strategy. In esophageal cancer the etiology differs depending on the histological type. In different communities and populations a diversified strategy is required. Colon cancer - although we are missing a specific individual target for primary prevention - is accessible for prevention by colonoscopic colorectal cancer screening for the early detection and removal of precursor lesions. In hepatocellular cancer prevention we have made an important step forward with new highly effective antiviral therapies. On the other side, non-alcoholic fatty liver disease has emerged as the most common 'liver concern' in industrialized countries by becoming the principal risk condition for hepatocellular cancer. Efforts to prevent obesity 
and metabolic syndrome, optimizing therapies in diabetes and surveillance strategies are becoming essential in this area.

You will miss the pancreas in this issue. Pancreatic cancer is still an unsolved problem for prevention and early detection, with the exception of a defined subset of patients at risk for genetically inherited cancer. We are awaiting new insights into the molecular mechanisms of pancreatic carcinogenesis and opportunities to successfully act in the prevention of this most 'dismal' malignant digestive disease.

The selected aspects for prevention of neoplastic digestive diseases presented in this issue should help in guiding our clinical management and in motivating efforts in basic, translational and clinical research to further help in preventing the numerous cases with malignancies of the gastrointestinal tract. 